\title{
Activation of Mitogen-Activated Protein Kinase/Extracellular Signal-Regulated Kinase in Hippocampal Circuitry Is Required for Consolidation and Reconsolidation of Recognition Memory
}

\author{
Áine Kelly, ${ }^{1}$ Serge Laroche, ${ }^{2}$ and Sabrina Davis ${ }^{2}$ \\ ${ }^{1}$ Trinity College Institute of Neuroscience, Department of Physiology, Trinity College, Dublin 2, Ireland, and 2Laboratoire de Neurobiologie de \\ l'Apprentissage, de la Mémoire et de la Communication, Centre National de la Recherche Scientifique, Unité Mixte de Recherche 8620, Université Paris Sud, \\ 91405 Orsay, France
}

Consolidation and reconsolidation of long-term memory have been shown to be dependent on the synthesis of new proteins, but the specific molecular mechanisms underlying these events remain to be elucidated. The mitogen-activated protein kinase (MAPK) pathway can trigger genomic responses in neurons, leading to changes in protein synthesis, and several studies have identified its pivotal role in synaptic plasticity and long-term memory formation. In this study, we analyze the involvement of this pathway in the consolidation and reconsolidation of long-term recognition memory, using an object recognition task. We show that inhibition of the MAPK pathway by intracerebroventricular injection of the MEK [MAPK/extracellular signal-regulated kinase (ERK)] inhibitor U0126 blocks consolidation of object recognition memory but does not affect short-term memory. Brain regions of the entorhinal cortex-hippocampal circuitry were analyzed for ERK activation, and it was shown that consolidation of recognition memory was associated with increased phosphorylation of ERK in the dentate gyrus and entorhinal cortex, although total expression of ERK was unchanged. We also report that inhibition of the MAPK pathway blocks reconsolidation of recognition memory, and this was shown to be dependent on reactivation of the memory trace by brief reexposure to the objects. In addition, reconsolidation of memory was associated with an increase in the phosphorylation of ERK in entorhinal cortex and CA1. In summary, our data show that the MAPK kinase pathway is required for both consolidation and reconsolidation of long-term recognition memory, and that this is associated with hyperphosphorylation of ERK in different subregions of the entorhinal cortex-hippocampal circuitry.

Key words: learning; reactivation; synaptic plasticity; dentate gyrus; CA1; entorhinal cortex

\section{Introduction}

Consolidation of newly acquired information is a necessary and critical process for the establishment of long-lasting memories. A long history of human and animal research has shown that, shortly after a learned event, initial memory traces are fragile and susceptible to disruption (McGaugh, 1966). The current consensus suggests that consolidation of the memory trace takes place at two different levels: the cellular and the systems levels. At the cellular level, early consolidation of certain hippocampaldependent memories requires activation of the genetic machinery and synthesis of new proteins (Abel and Lattal, 2001). At the systems level, when the trace is believed to be permanent, it is stored in cortical regions and is insensitive to hippocampal disruption (Marr, 1971; Eichenbaum et al., 1994; Squire and Alvarez, 1995).

Originally considered to be a unidirectional process, in which

Received Feb. 26, 2003; revised April 3, 2003; accepted April 7, 2003.

This work was supported by Grant RGY0152 from the Human Frontier Science Program to S.D. and the Health Research Board, Ireland.

Correspondence should be addressed to Dr. Sabrina Davis, Laboratoire de Neurobiologie de I'Apprentissage, de la Mémoire et de la Communication, Centre National de la Recherche Scientifique, Unité Mixte de Recherche 8620, Université Paris Sud, 91405 Orsay, France. E-mail: sabrina.davis@ibaic.u-psud.fr.

Copyright $\odot 2003$ Society for Neuroscience $\quad$ 0270-6474/03/235354-07\$15.00/0 the trace, once consolidated, was resistant to disruption, several studies have challenged this concept (Misanin et al., 1968; Mactutus et al., 1979; Judge and Quartermain, 1982). More recently, it has been demonstrated that a consolidated memory could be rendered labile and susceptible to disruption again when reactivated (Przybyslawski and Sara, 1997; Debiec et al., 2002; Milekic and Alberini, 2002). This suggests that, after reactivation of memory, an additional process, reconsolidation, is required for the trace to remain in long-term memory. It has been shown that both processes require protein synthesis (Nader et al., 2000; Taubenfeld et al., 2001; Debiec et al., 2002; Kida et al., 2002), but to date, only a few studies have identified specific mechanisms required, such as receptor (Przybyslawski and Sara, 1997; Przybyslawski et al., 1997) and cAMP response element-binding protein (CREB) (Kida et al., 2002) activation, whereas CCAAT/ enhancer-binding protein $(\mathrm{C} / \mathrm{EBP})$ seems necessary for consolidation but not reconsolidation (Taubenfeld et al., 2001). This raises important questions concerning which molecular mechanisms may be required for both processes, and which are not, and whether there is any form of task specificity.

Numerous studies have shown that activation of the MAP kinase (MAPK) pathway is critical for memory consolidation (Atkins et al., 1998; Berman et al., 1998; Blum et al., 1999; Selcher 
et al., 1999; Schafe et al., 2000). This pathway not only conveys signals from cell surface receptors to the nucleus, in which it participates in triggering the genomic response in neurons by activating immediate early genes (IEGs) (Davis et al., 2000; Waltereit et al., 2001), but also integrates signals from other transduction pathways. Given these characteristics, it has been considered to be a critical pathway that is implicated in long-lasting synaptic plasticity and the consolidation of long-lasting memories.

Our objective was to determine whether the MAP kinase pathway was implicated in both of the processes of consolidation and reconsolidation. To this end, we used an object recognition task that was adapted from human studies designed to test retrograde amnesia, targeting memory rather than learning (Ennaceur and Delacour, 1988). The task relies on rodents' innate propensity to explore novel objects, where having familiarized themselves with two or more objects, they can demonstrate that they remember the objects after a delay by showing preferential exploration of a novel object.

\section{Materials and Methods}

Animals. Sixty-four male Sprague Dawley rats (IFFA Credo, Saint Germain sur Arbresle, France) weighing between 350 and 450 gm were used. They were housed in pairs in a temperature-controlled vivarium with a $12 \mathrm{hr}$ light/dark cycle (lights on at 8:00 A.M.) and had access to food and water ad libitum. All of the experiments were performed in strict accordance with the recommendations of the European Union (86/609) and the French National Committee (87/848).

Surgical procedure and drug delivery. Rats were anesthetized with sodium pentobarbital $(60 \mathrm{mg} / \mathrm{kg})$ and supplemented throughout the surgical procedure as necessary. A single hole was drilled in the skull over the left ventricle (coordinates, bregma, $0.9 \mathrm{~mm}$; midline, $1.3 \mathrm{~mm}$ ), and a cannula (Phymep) was lowered slowly into the ventricle to a depth of 3.6 $\mathrm{mm}$ below the brain surface. A guide cannula was held in place and fixed to the skull with dental cement. The incision was closed with surgical staples, and topical antiseptic (exoceptoplix) was applied to the wound. Rats were given a $4 \mathrm{~d}$ course of terramycin antibiotic treatment and 7-10 $\mathrm{d}$ to fully recover before being tested.

The MAPK/extracellular signal-regulated kinase (ERK) kinase (MEK) inhibitor UO126 was dissolved in dimethylsulfoxide (DMSO) $(43.5 \%$ v/v) and artificial CSF (in mm: $120 \mathrm{NaCl}, 2.4 \mathrm{KCl}, 1.2 \mathrm{CaCl}, 1.2 \mathrm{MgCl}, 0.9$ $\mathrm{NaH}_{2} \mathrm{PO}_{4}, 1.4 \mathrm{Na}_{2} \mathrm{HPO}_{4}$, and 0.3 ascorbic acid) and stored at $-20^{\circ} \mathrm{C}$ until required. Injections of $4 \mu \mathrm{l}$ of either DMSO or UO126 $(1 \mu \mathrm{g} / \mu \mathrm{l})$ were made into the ventricle at a rate of $1 \mu \mathrm{l} / \mathrm{min} 40 \mathrm{~min}$ before rats were tested.

Object recognition task. The apparatus consisted of a black circular open field (diameter, $0.9 \mathrm{~m}$; height, $0.48 \mathrm{~m}$ ) placed in a room with somber lighting. All of the rats were handled daily for 1 week and habituated to the experimental apparatus, with 20 min of exploration in the absence of objects each day for $5 \mathrm{~d}$ before the experiment was performed. Objects were constructed from Lego, often incorporating a small piece of laboratory equipment. They were fixed to the floor of the open field, $15 \mathrm{~cm}$ from the walls. Objects were cleaned thoroughly between trials to ensure the absence of olfactory cues. The criteria for exploration were strictly based on active exploration, in which rats had to be touching the object with at least their noses. Measurement of the time spent exploring each object was recorded and expressed as a percentage of the total exploration time in seconds. Student's $t$ test and ANOVA were used to assess behavioral data.

Consolidation protocol. On day 1, rats were placed in the open field containing two different objects. They were given three 5 min periods in which to explore the objects with an intertrial interval of $5 \mathrm{~min}$. During the $5 \mathrm{~min}$ interval, rats were placed in a holding cage in the room. Either $10 \mathrm{~min}$ or $24 \mathrm{hr}$ later, one of the objects was randomly exchanged for a novel object, and rats were reintroduced into the open field for a single 5 min period, and the time spent exploring each object was recorded. To test whether activation of the MAP kinase pathway was necessary for recognition memory, 40 min before the sample phase on day 1 , rats were infused with either the MEK inhibitor UO126 or the vehicle solution DMSO. We verified that the MEK inhibitor substantially reduced constitutive phosphorylation of ERK in naive rats by $\sim 25 \%$ when injected at this time.

Reconsolidation protocol. In the sample phase (day 1), rats were exposed to two objects for three $5 \mathrm{~min}$ periods, as described above. Twentyfour hours later (day 2), rats were exposed to the same two sample objects for a single $5 \mathrm{~min}$ period to reactivate the memory trace. They were infused with either the MEK inhibitor UO126 or the vehicle solution DMSO 40 min before the reactivation session. On day 3, rats were reintroduced into the open field, in which one of the objects was exchanged for a novel object, and the time spent exploring each object was recorded. Another group of rats was injected with either UO126 or DMSO on day 2 but was not placed in the open field and was tested in the same manner on day 3.

To test whether MAPK/ERK phosphorylation occurred in structures of the temporal lobe during consolidation and reconsolidation, a group of noncannulated rats was killed $5 \mathrm{~min}$ after the three brief exposures to the two sample objects on day 1 , and another group was killed $10 \mathrm{~min}$ after the reactivation session on day 2. This timing was selected, because it approximately equates the time delay for ERK analysis after the start of reactivation to that after the middle of the learning phase. Control rats were placed in the open field in the absence of objects in each condition.

Preparation of cell lysates. Rats were killed by stunning and decapitation; brains were removed immediately, and the dorsal and ventral dentate gyrus, CA1, and the perirhinal and entorhinal cortices were dissected. Tissue samples were homogenized in lysis buffer $(250 \mu \mathrm{l} ; 50 \mathrm{~mm}$ $\mathrm{NaCl}, 50 \mathrm{~mm} \mathrm{NaF}, 30 \mathrm{~mm} \mathrm{NaPPi}, 10 \mathrm{~mm}$ Tris-HCl, 1 mм DTT, $500 \mu \mathrm{m}$ benzamidine, $100 \mu \mathrm{M} \mathrm{Na} \mathrm{VO}_{4}, 500 \mathrm{~nm}$ PMSF, $5 \mathrm{~nm}$ okadaic acid, 2.5 $\mu \mathrm{g} / \mathrm{ml}$ aprotinin, $2.5 \mu \mathrm{g} / \mathrm{ml}$ pepstatin, $2.5 \mu \mathrm{g} / \mathrm{ml}$ leupeptin, and $1 \% \mathrm{v} / \mathrm{v}$ Triton X-100), incubated on ice for $20 \mathrm{~min}$, and centrifuged at $13,000 \times$ $g$ for $20 \mathrm{~min}$ at $4^{\circ} \mathrm{C}$. Supernatants were removed and frozen at $-20^{\circ} \mathrm{C}$ for later analysis of phospho-ERK and total ERK.

Western immunoblotting. Cell lysates were assayed for protein content according to the method of Bradford (1976) and diluted to give equal protein concentrations. Samples were diluted 1:2 in sample buffer $(0.5$ mм Tris-HCl, pH 6.8, 10\% glycerol, $10 \%$ SDS, 5\% $\beta$-mercaptoethanol, and $0.05 \% \mathrm{w} / \mathrm{v}$ bromophenol blue) and boiled for $5 \mathrm{~min}$, and $10 \mu \mathrm{l}$ aliquots were loaded onto $10 \%$ SDS gels. Proteins were separated by application of a constant voltage of $200 \mathrm{~V}$ for $35 \mathrm{~min}$ and then transferred onto nitrocellulose membranes at a constant voltage of $100 \mathrm{~V}$ for $1 \mathrm{hr}$. After blocking in Tris-buffered saline (TBS)-Tween 20 ( $150 \mathrm{~mm} \mathrm{NaCl}, 50$ $\mathrm{mm}$ Tris- $\mathrm{HCl}$, and $0.05 \% \mathrm{v} / \mathrm{v}$ Tween $20, \mathrm{pH} 7.4$ ) containing bovine serum albumin (BSA) (4\% w/v), membranes were washed in TBS-Tween 20 and incubated in anti-phospho-ERK [1:2000 in TBS-Tween 20 containing BSA (2\%); Cell Signaling Technology, Beverly, MA] for $90 \mathrm{~min}$ at room temperature. Membranes were washed and incubated in a horseradish peroxidase-conjugated anti-rabbit IgG [1:2000 in TBS-Tween 20 containing BSA ( $2 \% \mathrm{w} / \mathrm{v})$; Amersham Biosciences, Little Chalfont, Buckinghamshire, UK] for $1 \mathrm{hr}$ at room temperature before being reacted with ECL solution (Amersham Biosciences), exposed to film for $1 \mathrm{hr}$, and developed by hand. Initial control experiments determined the optimal time of exposure to film, which was maintained throughout the experimental procedure. Protein bands were quantified using GeneSnap acquisition and GeneTools analysis software (GeneGenius Gel documentation and analysis system; Syngene, Cambridge, UK) to yield a figure in arbitrary units that represented the density of protein bands. To analyze the total ERK content of the samples, membranes were stripped of antibody by incubating in reblot plus solution (Chemicon, Temecula, CA), washed, and blocked in TBS-Tween 20 containing BSA (4\% v/v). No bands were detected in stripped blots incubated in secondary antibody alone, thus verifying the efficacy of the stripping protocol. Membranes were washed in TBS-Tween 20 and incubated in anti-ERK [1:3000 in TBS-Tween 20 containing BSA (2\% w/v); Ozyme, Saint Quentin Yvelines, France ] for $60 \mathrm{~min}$ at room temperature and then washed and incubated in a horseradish peroxidase-linked anti-rabbit IgG [1:3000 in TBS-Tween 20 containing BSA (2\% w/v); Amersham Biosciences] before being reacted with ECL solution (Amersham Biosciences). Blots were exposed to film for $1 \mathrm{hr}$ and developed by hand, and protein bands 

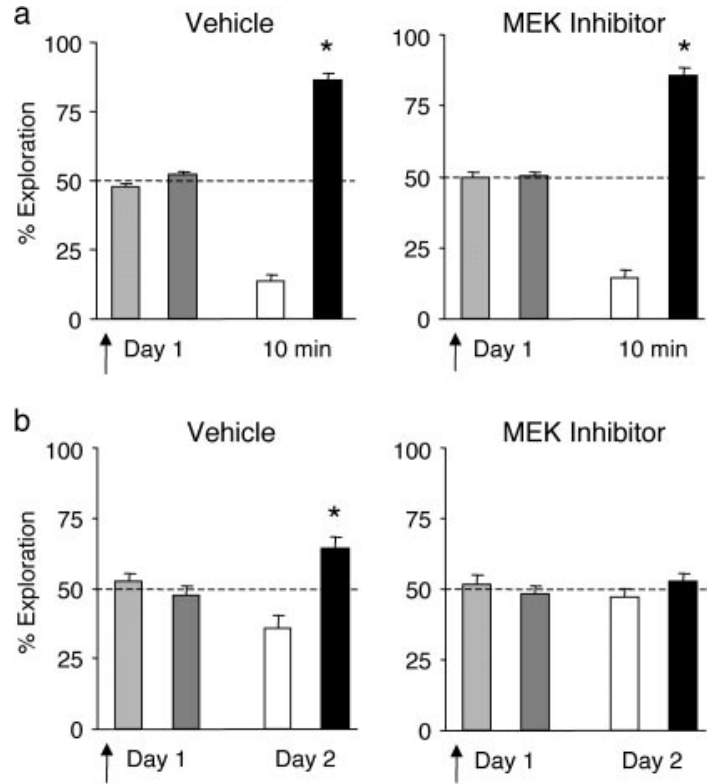

Figure 1. The effect of inhibition of MEK on consolidation of memory in object recognition. Arrows indicate injection of either a vehicle solution or the MEK inhibitor. Exploration of the objects in the sample phase are represented as light/dark gray bars. After the delay, exploration of the familiar object is represented by the white bar, and exploration of the novel object is represented by the black bar. $a$, Short-term retention. During the sample phase on day 1, rats spent an equivalent amount of time exploring the two objects. Exposure to a familiar and a novel object 10 min later resulted in significantly greater exploration of the novel object in both control and U0126-treated groups. $b$, Long-term retention. Both groups showed equal exploration of the two objects during the sample phase, but exposure to a familiar and a novel object $24 \mathrm{hr}$ later on day 2 resulted in a significantly greater exploration of the novel object in the control group but not in the U0126-treated group. Histograms represent mean time in exploration with SEMs. Dashed lines represent equal exploration of the objects, and asterisks represent a significant increase in exploration of the object.

were quantified as described above. Values presented here are means of data generated from at least three separate experiments. Because there was no change in the levels of total ERK1 and ERK2, values of phosphorylated ERK1/2 (pERK1/2) were normalized to the values of total ERK1 and ERK2, and ANOVA and Student's $t$ test were used for statistical analysis.

\section{Results}

Effect of MEK inhibition on learning and consolidation of memory

To test whether activation of the MAP kinase pathway was necessary for memory consolidation in the object recognition task, rats were injected intracerebroventricularly with the vehicle DMSO $(n=5)$ or the MEK inhibitor UO126 $(n=6) 40 \mathrm{~min}$ before the sample phase on day 1 . In this phase of the experiment, rats in both groups showed equal exploration of the sample objects (Fig. 1). The total time spent in exploration of objects did not differ between the two groups (all of the $p$ values were $>0.05$ ), suggesting that inhibition of ERK1/2 phosphorylation had no discernible effects on locomotor activity or the normal tendency for exploration of objects. When tested at a short interval of 10 min after the sample phase, both groups showed significantly greater exploration of the novel object compared with that of the familiar object ( $p<0.001$ for both groups) (Fig. 1a). The preference for the novel object in both groups indicates that inhibition of MEK has no effect on the ability of rats to learn about the objects and to express normal short-term recognition memory. In another experiment, rats were tested with a $24 \mathrm{hr}$ delay after the sample phase. As demonstrated in Figure $1 b$, on day 1 , there was no difference in the time spent exploring the two sample objects in either the DMSO-treated rats or the UO126-treated rats, nor in the total time spent in exploration $(p>0.05)$. On day 2 , control rats injected with DMSO $(n=7)$ showed preferential exploration of the novel object compared with that of the familiar object $(p<0.01)$. Although the percentage of exploration of the novel object in DMSO-treated rats was less at the $24 \mathrm{hr}$ delay compared with that at the 10 min delay, this was not significantly less than that of naive rats (mean of $62.55 \pm 1.68 \mathrm{sec} / 5 \mathrm{~min}$ ), suggesting that DMSO had no effect alone on recognition memory, and the difference was in all likelihood caused by a slight but normal decay of memory over the $24 \mathrm{hr}$ period. In contrast to the DMSO-treated rats, when UO126-treated rats $(n=6)$ were challenged with a novel and familiar object, they did not show preferential exploration of the novel object $(p>0.05)$. Therefore, these data suggest that activation of the MAP kinase pathway is necessary for long-term consolidation of recognition memory but not for short-term recognition.

\section{Activation of MAPK/ERK accompanies consolidation of memory}

We next used Western immunoblotting to examine ERK phosphorylation in corticohippocampal structures known to be implicated in recognition memory. Western immunoblotting analysis was performed on brain regions from a group of rats after exposure to the two objects during the sample phase on day 1 , a control group of rats that were placed in the open field in the absence of objects, and a group of naive rats. Rats exposed to the two objects showed equal exploration of both (means of $47.5 \pm$ 2.11 and $52.4 \pm 2.11 \mathrm{sec} / 5 \mathrm{~min} ; p>0.05)$. Expression of total ERK1 and total ERK2 did not differ between the groups in any of the structures examined (Fig. 2), suggesting that the levels of the proteins were not altered by exploration of the objects. Separate densitometric analysis of phosphorylation of ERK1 and ERK2, however, showed differential regulation in the brain regions examined (Fig. 2). In the dentate gyrus, there was a significant increase in the phosphorylation of ERK1 in the rats exposed to the objects compared with control $\left(F_{(1,24)}=5.94 ; p<0.05 ; n=\right.$ $17)$ but not with $\operatorname{ERK} 2\left(F_{(1,25)}=1.82 ; p>0.05\right)$, albeit there was a trend toward an increase in pERK2 (Fig. $2 a$ ). No difference was observed in pERK1 $(F<1)$ or pERK2 $(F<1)$ between the naive $(n=8)$ and control rats $(n=11)$. In CA1, as with the dentate gyrus, there was no significant difference in phosphorylation of pERK2 between the three groups; however, there was a significant increase in pERK1 $\left(F_{(2,33)}=3.75 ; p<0.05\right)$. Post hoc analysis showed that the difference was attributable to a significant increase in ERK1 in both of the rats exposed to the context alone and those exposed to the objects compared with the naive rats (Fig. $2 b$ ). In the entorhinal cortex, there was an increase in the phosphorylation of both ERK1 and ERK2 in rats exploring the objects (Fig. 2c), although it did not reach significance. No difference in either pERK1 $(F<1)$ or pERK2 $(F<1)$ was observed in the perirhinal cortex (Fig. $2 d$ ). In neither the dentate gyrus nor CA1 were there any changes in pERK1 or pERK2 in the ventral regions (data not shown). Finally, in a group of rats in which pERK1 and pERK2 were analyzed 25 min after the end of the sample phase, we observed no significant difference in the levels of pERKs in rats that explored the objects compared with either control rats or naive rats (all of the $p$ values were $>0.05$ ), suggesting a transient activation of ERK. These data suggest that exploration of objects is associated with hyperphosphorylation of ERK, mostly in the dentate gyrus and CA1 of the hippocampus and to a lesser extent in the entorhinal cortex, with hyperphosphoryla- 
a Dentate gyrus
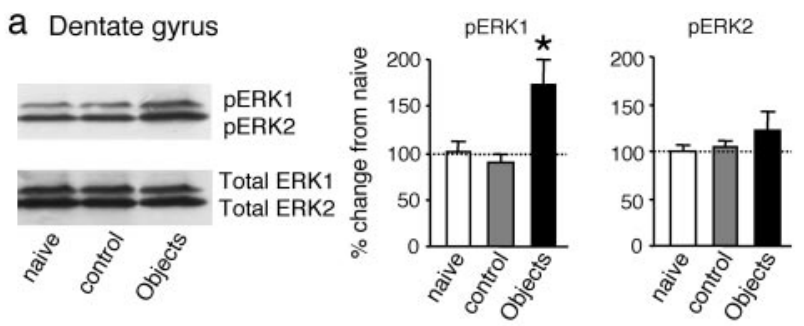

b CA1
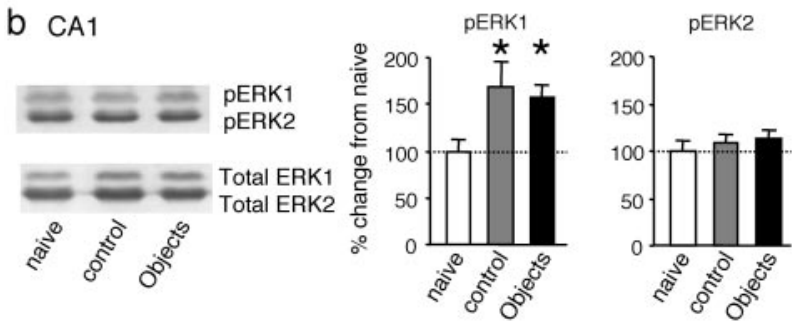

C Entorhinal cortex
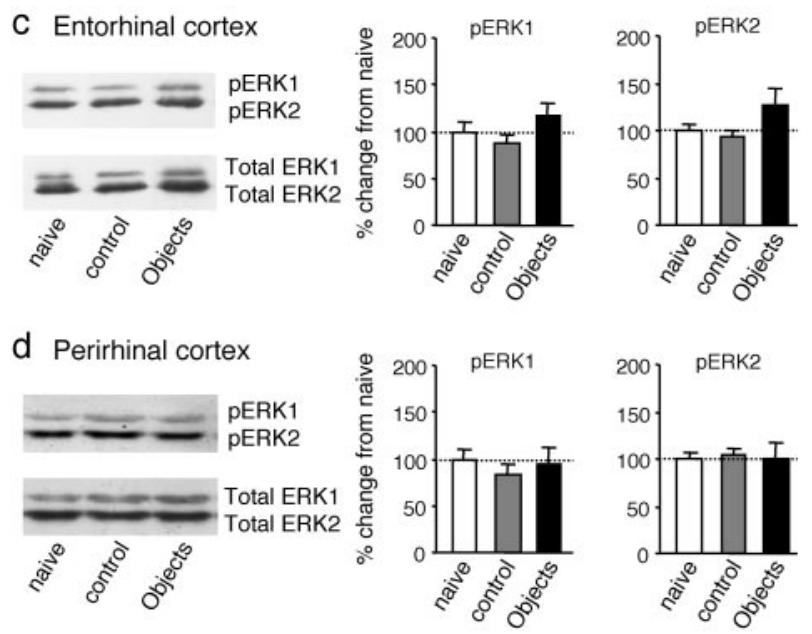

Figure 2. An increase in ERK phosphorylation is associated with memory consolidation. Sample Western blots for each brain region are presented to the left of the histograms. $a$ Histograms show significant hyperphosphorylation of ERK1 in rats exploring the objects compared with control and naive rats with no change in pERK2 in the dentate gyrus. $b$, In dorsal CA1, pERK1 was significantly increased in the control rats and those exploring the objects compared with naive rats, with no change in pERK2. c, In the entorhinal cortex, although it did not reach statistical significance, there was a substantial increase in phosphorylation of both ERK1 and ERK2. $d$, No change in the phosphorylation of ERK1 and ERK2 was observed in the perirhinal cortex. No change in total ERK1 and ERK2 was observed in any group in any of the brain structures, asillustrated in the sample Western blots. Dotted lines represent control levels of pERK, and asterisks represent significant increase in PERK.

tion of ERK1 playing a more prominent part in this process. In CA1, however, hyperphosphorylation of ERK1 was also found in the control group that spent time in the open field in the absence of objects, suggesting that ERK1 may be activated in response to environmental exploration. Thus, together with our results using the MEK inhibitor, our data suggest that the MAPK/ERK cascade is activated rapidly and transiently in certain regions of the entorhinal-hippocampal circuitry after exploration of objects and is necessary for the consolidation of long-term recognition memory.

\section{Effect of MEK inhibition on reconsolidation of memory}

Previous evidence has suggested that, in certain forms of memory, reconsolidation may require the same mechanisms as those involved in consolidation. Furthermore, it has been shown that
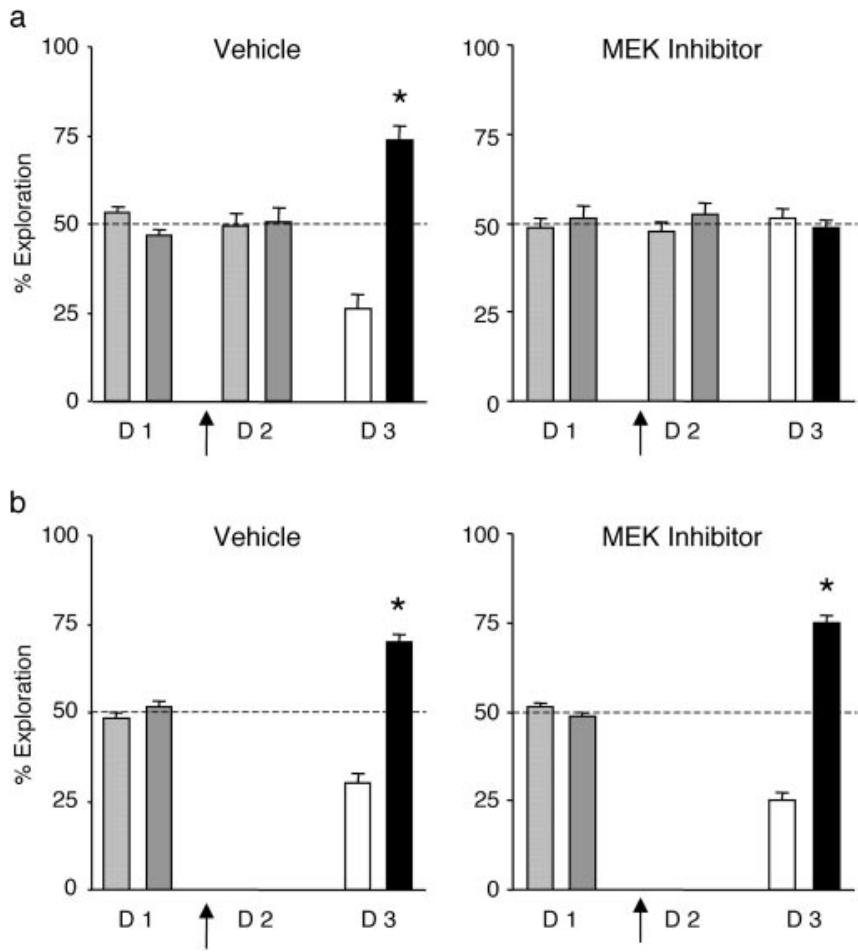

Figure 3. $a$, The effect of MEK inhibition on the reconsolidation of memory. Arrows indicate injection of DMSO or U0126 on day 2. Graphical representation is the same as in Figure 1. Rats spent an equal amount of time exploring the two objects on both day 1 (D 1; sample phase) and day 2 (D 2; reactivation). Exposure to a familiar and a novel object on day 3 (D 3) resulted in a significantly greater exploration of the novel object in the control group but not in the U0126treated group. $b$, Blockade of memory reconsolidation by MEK inhibition is dependent on reexposure to the objects. Both groups of rats spent an equal amount of time exploring the two objects on day 1. On day 2, rats received an injection of DMSO or U0126, as indicated by an arrow, but remained in the home cage. Exposure to a familiar and a novel object on day 3 resulted in a significantly greater exploration of the novel object in the control group in both control and U0126-treated groups. Dashed lines, SEMs, and asterisks are as referred to in Figure 1.

disruption of reconsolidation is contingent on reactivation of the memory trace. To test whether the MAP kinase pathway was necessary for reconsolidation of recognition memory, we first exposed rats to two objects in the sample phase on day 1. As shown in Figure $3 a$, there was no difference in time spent exploring each of the objects in either group of rats (both $p$ values were $>0.05$ ) or in the total time spent in exploration (DMSO, $71.56 \pm$ $9.88 \mathrm{sec}$; UO126, $69.6 \pm 8.24 \mathrm{sec} ; p>0.05)$. On day 2 , rats were briefly reexposed to the same object for one $5 \mathrm{~min}$ session to reactivate the memory trace. Forty minutes before this exposure period, rats were injected with either DMSO $(n=6)$ or UO126 $(n=7)$. Again, there was no significant difference in the percentage of time spent exploring objects in the DMSO-treated group $(p>0.05)$ or the UO126-treated group $(p>0.05)$, suggesting that the inhibitor had no effect on recall. In addition, there was no difference in the total time spent in exploration on the last session of the sample phase and the reactivation session $24 \mathrm{hr}$ later in either the control or experimental rats (both $p$ values were $>0.05)$. This suggests there was no discernible difference in the exploratory behavior between these two sessions. When the DMSO-treated rats were challenged with a familiar object and the novel object on day 3, they showed preferential exploration of the novel object ( $p<0.01$ ), as expected. In contrast, however, the UO126-treated group did not show a preference to the novel object; they equally explored each of the objects $(p>0.05)$. 
These results suggest that inhibition of the MAP kinase pathway during memory retrieval or reactivation of the trace results in impairment in the subsequent consolidation of recognition memory, suggesting an MAP kinase-dependent mechanism of reconsolidation.

\section{The effect of MEK inhibition on reconsolidation of memory is dependent on reactivation of the memory trace}

To test whether reexposure to the objects on day 2 was requisite for the impairment in reconsolidation induced by inhibiting the MAP kinase pathway, an additional group of rats was tested. Rats were exposed to the two objects on day 1 and injected on day 2 with DMSO $(n=6)$ or UO126 $(n=7)$ but remained in their home cages instead of being reexposed to the objects. On day 3 , both groups of rats were challenged with one familiar object and the novel object, as described previously. The results showed that, on days 1 and 2, all of the rats showed equal exploration of the objects (Fig. 3b). On day 3, both DMSO-treated and UO126treated rats showed significantly greater exploration of the novel object (both $p$ values were $<0.001$ ). Together with the data described above, this suggests that reexposure to the objects renders the memory trace fragile once more, and this is necessary for triggering an MAPK/ERK-dependent process of reconsolidation of recognition memory.

Activation of MAPK accompanies reconsolidation of memory The data presented thus far suggest that consolidation of recognition memory involves activation of the MAP kinase pathway and that inhibition of MEK blocks consolidation and reconsolidation of memory after reactivation of the memory trace. It is therefore likely that reactivation of an already consolidated memory trace activates the phosphorylation of ERK. To investigate this, two groups of rats were exposed to two objects on day 1 . On day 2 , rats in the control group $(n=6)$ were placed in the open field in the absence of objects, and the others $(n=6)$ were briefly exposed to the same two objects. Again, both groups showed an equal exploration of the objects on day 1 ( $p>0.05$; data not shown), as did the rats exposed to the same two objects on day 2 ( $p>0.05$; data not shown). Western immunoblotting was performed on tissue from the dorsal dentate gyrus and CA1, and the perirhinal and entorhinal cortices. We found no significant change in levels of phosphorylation of either ERK1 or ERK2 in the dentate gyrus (both $p$ values were $>0.05$ ) (Fig. $4 a$ ). In CA1 of the hippocampus, there was a significant increase in both pERK1 $(p<0.05)$ and pERK2 $(p<0.05)$ after reexposure to the objects (Fig. 4b). In addition, we also found a significant increase in phosphorylation of ERK1 in the entorhinal cortex $(p<0.05)$ but not in ERK2 $(p>0.05)$ (Fig. $4 c)$. There was no evidence of increased phosphorylation of either form of ERK in the perirhinal cortex (both $p$ values were $>0.05$ ) (Fig. $4 d$ ).

These data show that when rats are exposed to the same objects that they observed $24 \mathrm{hr}$ earlier during the sample phase, MAPK is hyperphosphorylated, but in parts of the corticohippocampal circuitry different from those observed in rats after the sample phase.

\section{Discussion}

The concept of cellular consolidation and reconsolidation of memories is based on experimental data showing that newly formed and reactivated but previously consolidated memories are susceptible to disruption by the inhibition of protein synthesis (Nader et al., 2000; Taubenfeld et al., 2001; Debiec et al., 2002; Kida et al., 2002). Although many of the mechanisms underlying a Dentate gyrus
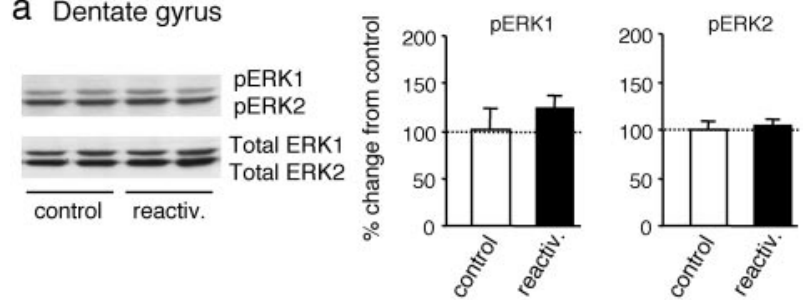

b CA1
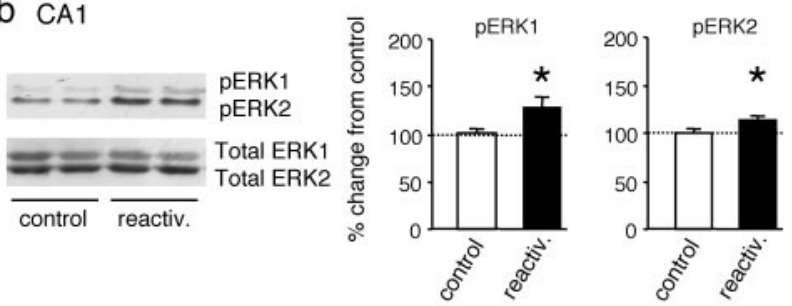

C Entorhinal cortex
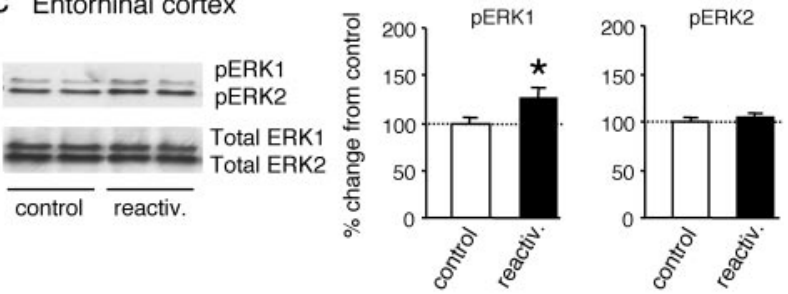

d Perirhinal cortex
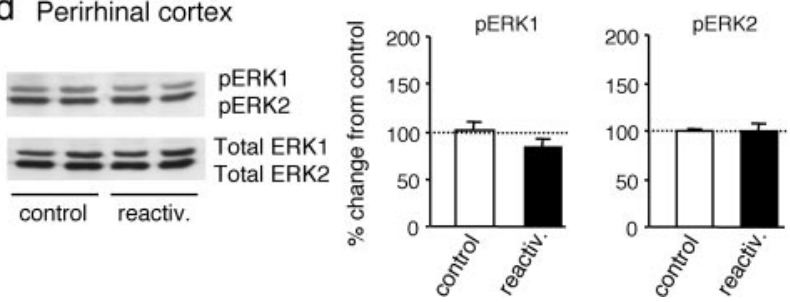

Figure 4. An increase in ERK phosphorylation is associated with memory reconsolidation. Sample Western blots for each brain region are presented to the left of the histograms. Densitometric analysis was conducted on rats that were reexposed to the objects on day 2 to reactivate the memory trace (reactiv.), or control rats. $a$, In the dorsal dentate gyrus, there was no significant increase in pERK1 or pERK2 in the reactivation group. $b$, In dorsal CA1, there was a significant increase in pERK1 and pERK2 in the reactivation group. $c$, In entorhinal cortex, phosphorylation of ERK1 was significantly increased after reactivation compared with controls. $d$, No change in phosphorylation of either isoform of ERK was observed in the perirhinal cortex. No change in total ERK1 and ERK2 was observed in any group in any of the brain structures, as illustrated in thesample Western blots. Dotted lines and asterisks are as referred to in Figure 2.

consolidation of memories have been well characterized, and numerous studies have implicated the need for the activation of different kinases (Abel and Lattal, 2001), to date, the mechanisms underlying reconsolidation are poorly understood. Here, we show that activation of the MAP kinase pathway is necessary for both of the processes of consolidation and reconsolidation of recognition memory and that rapid activation of MAPK/ERK occurs in the entorhinal-hippocampal circuitry in association with both processes.

We demonstrated the need for activation of the MAP kinase pathway for consolidation of recognition memory by blocking the activation of MAPK/ERK during the sample phase. We found that, although when tested at a short delay, rats are able to demonstrate memory of the objects they had explored previously by showing preferential exploration of a novel object, they are not able to do so when tested $24 \mathrm{hr}$ later, suggesting that activation of the MAP kinase pathway is necessary for the consolidation of memory for objects. These data on recognition memory are in 
keeping with several other reports showing that activation of this signaling cascade is necessary for consolidation of different forms of long-term memories (Brambilla et al., 1997; Atkins et al., 1998; Berman et al., 1998; Blum et al., 1999; Selcher et al., 1999; Walz et al., 1999, 2000).

We also showed differential phosphorylation of ERK in the entorhinal-hippocampal circuitry, confirming the implication of this signaling pathway in the processes of cellular consolidation. We found hyperphosphorylation of ERK1 in the dentate gyrus and to a lesser extent in the entorhinal cortex, which was specific to consolidation of information about the two objects. In CA1, we also found an increase in phospho-ERK1 after the exploration of the objects, but, in addition, it was also increased after exploring the open field in the absence of objects. Although it is well established that CA1 neurons are activated during exploratory behavior (O'Keefe and Nadel, 1978), the rats were well habituated to the open field, and therefore it is uncertain whether ERK1 phosphorylation in this region after the initial exposure to the objects is related to memory consolidation or is more sensitive to environmental stimulation. Because the rats were well habituated to the context before the sample phase, it is also possible that this hyperphosphorylation of ERK in CA1 in the control animals may reflect a mechanism of reconsolidation of the memory of the context.

We used the object recognition task, because it is exquisitely adapted to test memory, based on the rodent's natural propensity to explore novelty. Although studies have suggested that the perirhinal cortex may have a more critical role in the object recognition task than does the hippocampus (for review, see Mumby, 2001), we did not find hyperphosphorylation of ERK in this region. More recently, however, reports have suggested that the hippocampus is necessary for specific elements of the task, such as the spatial configuration of objects (Wan et al., 1999), the ability to remember episodes (Manns et al., 2003), and the temporal delay when testing memory (Clark et al., 2000). The delay for testing recognition memory appears to be a critical element, because rats with hippocampal lesions (Clark et al., 2000) and mice with CA1-specific deletions of CREB/ATF (activating transcription factor) (Pittenger et al., 2002) or the R1 subunit of the NMDA receptor (Rampon et al., 2000) are impaired in recognition memory when tested after a delay of $24 \mathrm{hr}$. Thus, our data showing activation of the MAP kinase signaling cascade in the entorhinal cortex-hippocampal circuitry in object recognition are in keeping with previous results. We do not, however, dismiss the possibility that activation of MAPK/ERK may occur in other brain structures or that the perirhinal cortex may be involved in recognition memory, although in an MAPK/ERK-independent manner.

The results from our second experiment show that activation of the MAP kinase pathway is necessary for reconsolidation of recognition memory. The process of reconsolidation is contingent on reactivation of the memory trace (in our experiments, the presentation of the two objects), which presumably renders the established trace labile once again. It is presumed that reactivation of the memory trace either reactivates the same cascades observed after consolidation or may activate different ones. We demonstrated the need for the activation of the MAP kinase pathway for reconsolidation, because when MAPK/ERK was inhibited during the reexposure period only, rats were unable to demonstrate intact memory $24 \mathrm{hr}$ later. The impairment in object recognition was not observed if rats were injected with the inhibitor and not reexposed to the objects, and, in addition, we observed an increase in pERK in areas of the entorhinal-hippocampal circuitry after the reexposure period. Thus, a brief period of reexposure to the objects reactivates the MAP kinase pathway, and its inhibition results in a failure to reconsolidate the memory trace.
Although we demonstrate that activation of the MAP kinase pathway is necessary for both consolidation and reconsolidation of recognition memory, our biochemical analysis shows that ERK activation occurs in different areas of the entorhinal cortex-hippocampal circuitry during the two processes. Whereas we found hyperphosphorylation of MAP kinase in association with both periods of consolidation and reconsolidation in the entorhinal cortex, in the dentate gyrus, ERK was hyperphosphorylated only in relation to consolidation. Furthermore, in CA1, whereas hyperphosphorylation of MAP kinase did not appear to have specificity after the initial exposure to the objects, it was specifically associated to the reactivation of the memory for the objects. Our results suggest that different subregions are activated during the consolidation of newly acquired information and during retrieval of already consolidated information. Although we cannot completely rule out the possibility that the differential regulation of ERK may be linked to a slightly different behavior during the initial consolidation phase and the reexposure phase (Nader, 2003), our analysis showed that the rats spent a similar amount of time in exploration in both the last session of the sample phase and the reactivation phase. These results highlight an entorhinaldentate gyrus and possibly CA1 circuit as part of a network implicated in the consolidation of recognition memory and a more restricted entorhinal cortex-CA1 circuit implicated in reconsolidation after retrieval of the memory. The particular sensitivity of CA1 to retrieval has also been demonstrated in experiments showing upregulation of the expression of the immediate early gene zif268 in contextual fear conditioning (Hall et al., 2000).

Our data on ERK and that of Kida et al. (2002) on CREB show that both proteins are necessary for the two memory processes, albeit the memories are different (recognition memory vs fearassociated memory). Because CREB is a downstream target of ERK (Sweatt, 2001; Lonze and Ginty, 2002), it suggests that a similar signaling cascade leading to activation of IEGs and gene transcription is activated in both consolidation and reconsolidation of memory. It is not the case, however, that all of the proteins implicated in the consolidation of memories are necessarily involved in its reconsolidation. Taubenfeld et al. (2001) have shown that the transcription factor $\mathrm{C} / \mathrm{EBP} \beta$, which is also activated by ERK (Hu et al., 2001), is necessary for consolidation, but not reconsolidation of inhibitory avoidance memories. This does not, however, suggest that the same mechanisms are not implicated in reconsolidation, because they may be activated in other parts of a network associated with encoding different memories, and, in addition, there are data to suggest a degree of task and structure specificity. For example, inhibitors of protein synthesis in the nucleus accumbens prevent the consolidation of newly acquired appetitive instrumental memories but not the reconsolidation of them (Hernandez et al., 2002), and, when injected into the hippocampus, the inhibitors prevent consolidation of inhibitory avoidance but not the reconsolidation of it (Taubenfeld et al., 2001). An additional argument comes from studies of regulated expression of zif268 in fear conditioning. Hall and colleagues showed that zif268 is not regulated in CA1 after contextual fear conditioning (Hall et al., 2001), but it is overexpressed in CA1 (Hall et al., 2001) and in other structures, such as the frontal cortex and nucleus accumbens after retrieval of the fear memories (Thomas et al., 2002). Together, the results suggest that the processes of reconsolidation may be dependent on an intricate combination of the types of memories encoded and the brain regions and cellular cascades that are involved.

In summary, our data on recognition memory have shown two important aspects associated with the dynamics of memory process- 
ing: it undergoes a process of reconsolidation, and the MAPK kinase pathway is required for both consolidation and reconsolidation of a previously consolidated memory when recalled. In parallel, we observed hyperphosphorylation of ERK in the entorhinal cortex-hippocampal circuitry in both processes of consolidation and reconsolidation, although in different subregions of this circuitry. Given the known role of ERK phosphorylation in mechanisms underlying synaptic plasticity (Sweatt, 2001), these findings suggest that there is not a complete overlap in the neuronal circuits undergoing synaptic changes during consolidation and reconsolidation after recall. Whether the MAP kinase pathway is activated each time the memory trace is reactivated and whether its reactivation is specific to recognition memory or would constitute an all-purpose mechanism for memory stabilization remain open questions. It is possible that, because recognition memory is widely viewed as having an episodic or recollective component, it may well require reconsolidation every time the memory is recalled.

\section{References}

Abel T, Lattal KM (2001) Molecular mechanisms of memory acquisition, consolidation and retrieval. Curr Opin Neurobiol 11:180-187.

Atkins CM, Selcher JC, Petraitis JJ, Trzaskos JM, Sweatt JD (1998) The MAPK cascade is required for mammalian associative learning. Nat Neurosci 1:602-609.

Berman DE, Hazvi S, Rosenblum K, Seger R, Dudai Y (1998) Specific and differential activation of mitogen-activated protein kinase cascades by unfamiliar taste in the insular cortex of the behaving rat. J Neurosci 18:10037-10044.

Blum S, Moore AN, Adams F, Dash PK (1999) A mitogen-activated protein kinase cascade in the CA1/CA2 subfield of the dorsal hippocampus is essential for long-term spatial learning. J Neurosci 19:3535-3544.

Bradford M (1976) A rapid and sensitive method for the quantitation of microgram quantities of protein utilising the principle of protein dye binding. Anal Biochem 72:248-254.

Brambilla R, Gnesutta N, Minichiello L, White G, Roylance AJ, Herron CE, Ramsey M, Wolfer DP, Cestari V, Rossi-Amaud C, Grant SG, Chapman PF, Lipp HP, Sturani E, Klein R (1997) A role for the Ras signalling pathway in synaptic transmission and long-term memory. Nature 390:281-286.

Clark RE, Zola SM, Squire LR (2000) Impaired recognition memory in rats after damage to the hippocampus. J Neurosci 20:8853-8860.

Davis S, Vanhoutte P, Pages C, Caboche J, Laroche S (2000) The MAPK/ ERK cascade targets both Elk-1 and cAMP response element-binding protein to control long-term potentiation-dependent gene expression in the dentate gyrus in vivo. J Neurosci 20:4563-4572.

Debiec J, LeDoux JE, Nader K (2002) Cellular and systems reconsolidation in the hippocampus. Neuron 36:527-538.

Eichenbaum H, Otto T, Cohen NJ (1994) Two functional components of the hippocampal memory system. Behav Brain Sci 17:449-518.

Ennaceur A, Delacour J (1988) A new one-trial test for neurobiological studies of memory in rats. I. Behavioural data. Behav Brain Res 31:47-59.

Hall J, Thomas KL, Everitt BJ (2000) Rapid and selective induction of BDNF expression in the hippocampus during contextual fear conditioning. Nat Neurosci 3:533-535.

Hall J, Thomas KL, Everitt BJ (2001) Cellular imaging of zif268 expression in the hippocampus and amygdala during contextual and cued fear memory retrieval: selective activation of hippocampal CA1 neurons during the recall of contextual memories. J Neurosci 21:2186-2193.

Hernandez PJ, Sadeghian K, Kelley AE (2002) Early consolidation of instrumental learning requires protein synthesis in the nucleus accumbens. Nat Neurosci 5:1327-1331.

Hu J, Roy SK, Shapiro PS, Rodig SR, Reddy SP, Platanias LC, Schreiber RD, Kalvakolanu DV (2001) ERK1 and ERK2 activate CCAAAT/enhancerbinding protein- $\beta$-dependent gene transcription in response to interferon- $\gamma$. J Biol Chem 276:287-297.

Judge ME, Quartermain D (1982) Characteristics of retrograde amnesia following reactivation of memory in mice. Physiol Behav 28:585-590.

Kida S, Josselyn SA, de Ortiz SP, Kogan JH, Chevere I, Masushige S, Silva AJ (2002) CREB is required for the stability of new and reactivated fear memories. Nat Neurosci 5:348-355.
Lonze BE, Ginty DD (2002) Function and regulation of CREB family transcription factors in the nervous system. Neuron 35:605-623.

Mactutus CF, Riccio DC, Ferek JM (1979) Retrograde amnesia for old (reactivated) memory: some anomalous characteristics. Science 204:1319-1320.

Manns JR, Hopkins RO, Reed JM, Kitchener EG, Squire LR (2003) Recognition memory and the human hippocampus. Neuron 37:171-180.

Marr D (1971) Simple memory: a theory for archicortex. Philos Trans R Soc Lond B Biol Sci 262:23-81.

McGaugh JL (1966) Time-dependent processes in memory storage. Science 153:1351-1358

Milekic MH, Alberini CM (2002) Temporally graded requirement for protein synthesis following memory reactivation. Neuron 36:521-525.

Misanin JR, Miller RR, Lewis DJ (1968) Retrograde amnesia produced by electroconvulsive shock after reactivation of a consolidated memory trace. Science 160:203-204.

Mumby DG (2001) Perspectives on object-recognition memory following hippocampal damage: lessons from studies in rats. Behav Brain Res 127:159-181.

Nader K (2003) Memory traces unbound. Trends Neurosci 26:65-72.

Nader K, Schafe GE, LeDoux JE (2000) Fear memories require protein synthesis in the amygdala for reconsolidation after retrieval. Nature 406:722-726.

O'Keefe J, Nadel L (1978) The hippocampus as a cognitive map. London: Oxford UP.

Pittenger C, Huang YY, Paletzki RF, Bourtchouladze R, Scanlin H, Vronskaya S, Kandel ER (2002) Reversible inhibition of CREB/ATF transcription factors in region CA1 of the dorsal hippocampus disrupts hippocampusdependent spatial memory. Neuron 34:447-462.

Przybyslawski J, Sara SJ (1997) Reconsolidation of memory after its reactivation. Behav Brain Res 84:241-246.

Przybyslawski J, Roullet P, Sara SJ (1997) Attenuation of emotional and nonemotional memories after their reactivation: role of $\beta$ adrenergic receptors. J Neurosci 19:6623-6628.

Rampon C, Ya-Ping T, Goodhouse J, Shimitzu E, Kyin M, Tsien JZ (2000) Enrichment induces structural changes and recovery from nonspatial memory deficits in CA1 NMDA1-knockout mice. Nat Neurosci 3:238-244.

Schafe GE, Atkins CM, Swank MW, Bauer EP, Sweatt JD, LeDoux JE (2000) Activation of ERK/MAP kinase in the amygdala is required for memory consolidation of Pavlovian fear conditioning. J Neurosci 20:8177-8187.

Selcher JC, Atkins CM, Traskos JM, Paylor R, Sweatt JD (1999) A necessity for MAP kinase activation in mammalian spatial learning. Learn Mem 6:478-490.

Squire LR, Alvarez P (1995) Retrograde amnesia and memory consolidation: a neurobiological perspective. Curr Opin Neurobiol 5:169-177.

Sweatt JD (2001) The neuronal MAP kinase cascade: a biochemical signal integration system subserving synaptic plasticity and memory. J Neurochem 76:1-10.

Taubenfeld SM, Milekic MH, Monti B, Alberini CM (2001) The consolidation of new but not reactivated memory requires hippocampal C/EBP $\beta$. Nat Neurosci 4:813-818.

Thomas KL, Hall J, Everitt BJ (2002) Cellular imaging with zif268 expression in the rat nucleus accumbens and the frontal cortex further dissociated the neural pathways activated following the retrieval of contextual and cued memory. Eur J Neurosci 16:1789-1796.

Waltereit R, Dammermann B, Wulff P, Scafidi J, Staubli U, Kauselmann G, Bundman M, Kuhl D (2001) Arg3.1/Arc mRNA induction by $\mathrm{Ca}^{2+}$ and cAMP requires protein kinase $\mathrm{A}$ and mitogen-activated protein kinase/ extracellular regulated kinase activation. J Neurosci 21:5484-5493.

Walz R, Roesler R, Quevedo J, Rockenback IC, Amaral OB, Vianna MR, Lenz G, Medina JH, Izquierdo I (1999) Dose-dependent impairment of inhibitory avoidance retention in rats by immediate post-training infusion of a mitogen-activated protein kinase kinase inhibitor into cortical structures. Behav Brain Res 105:219-223.

Walz R, Roesler R, Quevedo J, Sant'Anna MK, Madruga M, Rodrigues C, Gottfried C, Medina JH, Izquierdo I (2000) Time-dependent impairment of inhibitory avoidance retention in rats by posttraining infusion of a mitogen-activated protein kinase kinase into cortical and limbic structures. Neurobiol Learn Mem 73:11-20.

Wan H, Aggleton JP, Brown MW (1999) Different contributions of the hippocampus and perirhinal cortex to recognition memory. J Neurosci 19: $1142-1148$ 